\title{
Effects of age, gender and educational background on strength of motivation for medical school
}

\author{
Rashmi Kusurkar · Cas Kruitwagen · Olle ten Cate · Gerda Croiset
}

Received: 2 July 2009/Accepted: 6 September 2009/Published online: 23 September 2009

(C) The Author(s) 2009. This article is published with open access at Springerlink.com

\begin{abstract}
The aim of this study was to determine the effects of selection, educational background, age and gender on strength of motivation to attend and pursue medical school. Graduate entry (GE) medical students (having Bachelor's degree in Life Sciences or related field) and Non-Graduate Entry (NGE) medical students (having only completed high school), were asked to fill out the Strength of Motivation for Medical School (SMMS) questionnaire at the start of medical school. The questionnaire measures the willingness of the medical students to pursue medical education even in the face of difficulty and sacrifice. GE students $(59.64 \pm 7.30)$ had higher strength of motivation as compared to NGE students $(55.26 \pm 8.33)$, so did females $(57.05 \pm 8.28)$ as compared to males $(54.30 \pm 8.08) .7 .9 \%$ of the variance in the SMMS scores could be explained with the help of a linear regression model with age, gender and educational background/selection as predictor variables. Age was the single largest predictor. Maturity, taking developmental differences between sexes into account, was used as a predictor to correct for differences in the maturation of males and females. Still, the gender differences prevailed, though they were reduced. Pre-entrance educational background and selection also predicted the strength of motivation, but the effect of the two was confounded. Strength of motivation appears to be a dynamic entity, changing primarily with age and maturity and to a small extent with gender and experience.
\end{abstract}

Keywords Age $\cdot$ Gender $\cdot$ Graduate entry $\cdot$ Maturity $\cdot$ Medical school · Medical students · Motivation

R. Kusurkar · G. Croiset

Rudolf Magnus Institute of Neuroscience, UMC Utrecht, Utrecht, The Netherlands

C. Kruitwagen

Julius Center, UMC Utrecht, Utrecht, The Netherlands

R. Kusurkar $(\bowtie) \cdot$ O. ten Cate $\cdot$ G. Croiset

Center for Research and Development of Education, UMC Utrecht, 3584 CG Utrecht, The Netherlands e-mail: R.Kusurkar@umcutrecht.nl; rashmi.kusurkar@gmail.com 


\section{Introduction}

Medical students are known to be highly motivated students, if measured by drop-out rate and time investment and compared with other students in higher education (VSNU 2008; QANU Report 2004). Strength of this motivation can be seen as an independent variable, predicting behaviour, but also as a dependent variable, affected by experiences in the past. Two fairly recent phenomena in undergraduate medical education may influence this strength, and indirectly its impact on academic success. One is the worldwide increase in the number of female students, who within a short period of time, often came to outnumber male medical students (Allen 2005; Bickel 2001; Heiligers and Hingstman 2000, Phillips and Austin 2009; Velden et al. 2008). The other is the enrolment of students with a graduate background when they start medical school. North America has employed this two-cycle model for a long time as the only curriculum structure, but in Europe and Australia, the admission of students who have graduated academically, into a shortened medical course or in a higher program year of a regular course, is relatively new (Prideaux and McCrorie 2004; ten Cate 2007). So, in most countries, students are admitted into medical school at the age of 17-18 years, directly after high school, whereas in North America and Canada students are admitted to medical school around the age of 23 years (Baum and Axtell 2005). Gender, academic background and maturity may well affect how motivated students are to pursue medical school, but the extent of these effects is not known.

There is reason to believe that younger students are less motivated for medicine than older students. In a study in Australia, it was found that only 52\% of newly admitted medical students actually had the ambition to enter medicine, $19 \%$ entered due to interest in Biology, $13 \%$ entered due to parental pressure and $12 \%$ entered because they obtained high marks, but had no idea of their career and $4 \%$ could not give any specific reason (Marley and Carman 1999). Studies done with a view to find out whether medical students are aware of the responsibilities they are choosing for have shown that students in their first year of medical school are not very familiar with the varied aspects and responsibilities of the medical profession. (Underwood et al. 1990; Marley and Carman 1999; Nieuwhof et al. 2005). The conceptions of first year medical students about the medical profession are often limited to the social aspects. Their general understanding is about only two activities; talking with patients and acting in the operation room (Nieuwhof et al. 2005). Many students have an almost total lack of knowledge about the career structures and working hours when they start (Underwood et al. 1990). This does not necessarily affect their strength of motivation for medical school, but the relationship between academic motivation and the validity of the image of the profession of these students is also not known.

If age and gender affect aptitude for medical school, this could be due to maturity being related to gender at ages between 14 and 20 years (Westenberg 2008) and brain development (Jolles et al. 2006; Pujol et al. 1993) and maturation (Jolles et al. 2006; Westenberg 2008), continuing up to the age of 24 years or third decade of life. Males can be considered to lag behind females by 2 years in physical maturation (Reed and Stuart 1959; Marshall and Tanner 1970; Tanner et al. 1976). There is evidence that the maturation of the brains of males lag behind the brains of females of corresponding age by 3 years, leading to a lag of 3 years in the emotional maturation of the males (Westenberg 2008). The psychosocial maturation and maturation of judgement also show a steady progress up to the age of 24 years (Steinberg and Cauffman 1996). In a study on medical school applicants in the United States, females were found to have significantly higher emotional 
intelligence (EI) as compared to males; the dimensions of the EI being maturity, compassion, morality, sociability, calm disposition (Carrothers et al. 2000).

Moreover, there is the belief that at the age of 17 years, the brain is not yet mature enough to make significant career choices or it could make risky choices (Steinberg 2005), hence there are many students dropping off or exchanging study (Westenberg 2008). This aspect of age at entry related to complex relations between gender and maturity and academic background has not been studied before in medical students.

The studies done on motivation of medical students have always assessed the quality or type of motivation. A student may have a good or desirable quality of motivation, but his level or strength of motivation may not be optimal (Nieuwhof et al. 2004). We were interested in knowing, measuring and understanding the strength of motivation. After reviewing the literature in search for a scale which measures the strength of motivation, only one questionnaire was found i.e. the SMMS (Strength of Motivation for Medical School) questionnaire (Nieuwhof et al. 2004). To the best of our knowledge, there is only one other study (Hulsman et al. 2007) which has investigated strength of motivation in medical students, using the same questionnaire, and it investigates the relation between motivation and performance.

The aim of the study was to investigate what difference educational background, preentrance selection, age and gender would make on motivation of students choosing for medical school.

The Netherlands is a suitable country to compare the effects of selection versus nonselection, as the country employs a weighted lottery selection procedure for most of the medical students admitted to the traditional six-year course of medicine. Moreover, at the University Medical Center Utrecht, there also exists a four-year graduate entry course in medicine, which selects students who have a bachelor's diploma in life sciences or a related field and have to go through a selection procedure. We will call these students the Graduate Entry students (GE students). The six-year course students are admitted in to medical course after high school diploma, i.e. they do not have a bachelor's degree; hence we will call them Non-Graduate Entry students (NGE students). Though these groups were not experimentally made, they were available for the research as convenience samples. This presented a unique opportunity to compare groups that were selected and not selected and also study the effect of age, gender, maturity and educational background on the motivation to pursue medical study in a similar medical education environment. The research questions for the study were:

1. Do GE medical students have stronger motivation for medical school as compared to NGE students?

2. What is the contribution of the factors of age, gender, educational background and preentrance selection towards strength of motivation for medical school?

\section{Methods}

\section{Subjects}

The subjects were students joining either one of the two medical courses: (1) students from the NGE course, batches 2006-2007, 2007-2008 ( $\mathrm{N}=540)$ and (2) students from the GE course, batches 2006-2007, 2007-2008, 2008-2009 ( $\mathrm{N}=153$ ). 
In all cohorts, the questionnaire was administered within three weeks of the beginning of medical school. The response rate for the students present on the particular day that the questionnaire was administered was $100 \%$. The total number of NGE students admitted during this period was 620 ; hence the response rate was $87.1 \%$. Most remaining subjects were not present because they had late enrolment and a few were not present that day for other reasons. The total number of GE students admitted was 161; hence the response rate was $95.0 \%$. For $20 \mathrm{NGE}$ students and $6 \mathrm{GE}$ students there were missing data; therefore they were excluded from the analysis (age was missing for 12 NGE females and 7 NGE males and gender was missing for one. Age and gender were missing for 6 GE students). Twelve NGE students held a bachelor's degree and nine GE students did not, hence were excluded from the analysis. Two GE students did not have a bachelor's degree, but had more than three years of biomedical education hence they were included in the analysis.

Instrument

The instrument used for determining the strength of motivation was the Strength of Motivation for Medical School (SMMS) questionnaire (Nieuwhof et al. 2004). The authors have defined strength of motivation for medical school as "the applicant's or student's readiness to start and continue medical training regardless of sacrifices, setbacks, misfortune or disappointing perspectives". The questionnaire contains sixteen items that are scored on a Likert point scale of 5, ranging from "Strongly Disagree" to "Strongly Agree". The minimum and maximum possible scores are 16 and 80 respectively. The higher the score, the greater is the strength of motivation. Nieuwhof et al. report favourable psychometric properties for this questionnaire; Cronbach's alpha for reliability of the scale was stated to be 0.79 and the test-retest reliability after 6 months period was 0.71 . The validity was examined by Nieuwhof within the framework of prototypicality approach, i.e. validation was done by asking peers or expert judges (potential applicants and parents) to assess the prototypicality of the constructed items for motivation. The correlation of the judges' scores and the medical students' scores, given the small numbers, seems to be satisfactory. Construct validity was studied by Nieuwhof in a group of potential applicants to medical school in two ways: Pearson correlation of SMMS scores with 'Ambivalence towards studying' for medical school was found to be negative $(-0.28)$; Spearman correlation between SMMS score and 'determination to apply for medical school' was found to be positive (0.65).

The SMMS questionnaire was administered to medical students around the same time of the medical course in our study as compared to Nieuwhof's study, also within the same institute. The scores were in line with the scores recorded by Nieuwhof, (Students admitted with lottery $=56.7 \pm 8.1$, Students admitted with Selection Procedure $=60.4 \pm 6.6$ ), though the total respondents in her study were much lesser (Nieuwhof et al. 2004).

\section{Conditions}

The subjects were not randomly assigned to the two groups, but were rather readily available.

The groups differed in the following aspects:

1. The GE students were older than the NGE students, their mean ages being 23.3 years and 18.3 years respectively. 
2. The GE students held a Bachelor's degree in Life/Health Sciences or Biomedicine, the NGE students held only a high school diploma.

3. The GE students were selected through a pre-entrance selection procedure, which was designed to select students who would be suitable for following the curriculum of the 4 year course. The NGE students were admitted through weighted lottery selection.

Therefore, there were differences in age, educational background and selection of the students between the two groups.

\section{Procedure}

At the end of a didactic lecture, the subjects were asked to fill out the SMMS questionnaire after informed consent. The participation was voluntary. The data obtained was accessible only to the researcher and anonymity was ensured.

\section{Statistical analysis}

For the nominal variable, gender, the Pearson's $\chi^{2}$ test was used to determine whether gender and background are independent.

For the continuous variable, the SMMS score, Student's $t$ test was used, to study differences between the two backgrounds and between the two genders.

For every statistically significant result, Cohen's effect size of the difference was determined.

SMMS scores were analyzed via multiple regression analysis to assess the effect of age, gender, maturity and background/pre-entrance selection.

Males mature later than the females. Since we wanted to investigate whether there was a gender difference, independently of maturity, a simple mathematical transformation of the age of the males was carried out to establish a new variable called maturity.

The assumptions for transforming the variable age into maturity in the present study were:

1. At the age of 24 years, males and females have completed their maturation process. (Steinberg and Cauffman 1996).

2. But at the age of 18 years, the males are three years behind the females (Westenberg 2008).

3. The "catch-up" can be modeled as a quadratic function, meaning that the amount of catching up decreases as the difference in maturity becomes smaller.

Thus, the males were made younger by three or less years so that their SMMS score was comparable to females younger than them by three years or less.

\section{Results}

The SMMS questionnaire had sufficient reliability, with a Cronbach's alpha of 0.735 for the GE group and 0.783 for the NGE group.

The two groups were not significantly different in gender (Pearson's $\chi^{2}=0.463$, df $=1, P=0.496$ ) (Table 1$)$.

The first step was to determine whether there was a difference in the strength of motivation between the NGE and the GE group. 
Table 1 Distribution of gender in the two groups

$\chi^{2}=0.463, \mathrm{df}=1, P=0.496$

Table 2 SMMS scores of NGE and GE students

$\mathrm{t}=-5.618, P=0.000$

\begin{tabular}{lrcc}
\hline Group & Males & Females & Total \\
\hline Non graduate entry & 154 & 354 & 508 \\
Graduate entry & 46 & 92 & 138 \\
Total & 200 & 446 & 646 \\
\hline
\end{tabular}

\begin{tabular}{llll}
\hline Group & N & Mean \pm SD & Mean age in years \\
\hline Non graduate entry & 508 & $55.26 \pm 8.33$ & 18.3 \\
Graduate entry & 138 & $59.64 \pm 7.30$ & 23.3 \\
\hline
\end{tabular}

Results depicted in Table 2, $\mathrm{t}=-5.618, P=0.000$, show that this difference was statistically significant and Cohen's effect size for this difference was 0.559 , i.e. more than a medium effect.

The second step was to determine whether there was a difference in the strength of motivation between males and females.

There was a significant difference between the scores of males and females (Table 3 and 4): NGE group, $\mathrm{t}=-3.684, P=0.000$, Cohen's effect size $=0.360$ i.e. between small and medium effect and GE group, $\mathrm{t}=-1.992, P=0.048$, Cohen's effect size of 0.351 i.e. between small and medium effect.

In the third step, since there was a large variation in the ages of students within and between both the programmes, the correlation of SMMS score with the age was investigated. The Pearson's correlation of SMMS score with age in the GE students group was 0.009 (not significant, Fig. 1) and in the NGE students group was 0.122 (significant, Fig. 2).

Since the GE group students were older, it was important to determine whether the higher motivation seen in them was due to the effect of age or due to the difference in the educational background or pre-entrance selection or both. We carried out a multiple regression analyses to determine the contribution of age, gender, background/pre-entrance selection, both with age as a variable (model 1) and with maturity instead as a variable (model 2) in Tables 5 and 6.

This showed that the Model 1, containing only Age, explained 3.9\% of the variance in the SMMS scores. By adding Gender, another $2.8 \%$ of the variance could be explained. On further adding Educational background as a variable, the model could explain an extra

Table 3 SMMS scores of male and female medical students, groups combined

\begin{tabular}{lll}
\hline Gender & $\mathrm{N}$ & Mean \pm SD \\
\hline Males & 200 & $54.30 \pm 8.08$ \\
Females & 446 & $57.05 \pm 8.28$ \\
\hline
\end{tabular}

$\mathrm{t}=3.927, P=0.000$

Table 4 SMMS scores of male and female medical students, separate groups

\begin{tabular}{|c|c|c|c|c|}
\hline Group & Males & Females & $\mathrm{t}$ & $P$ \\
\hline Non-graduate entry & $53.23 \pm 7.84$ & $56.15 \pm 8.39$ & -3.783 & 0.000 \\
\hline Graduate entry & $57.91 \pm 7.93$ & $60.51 \pm 6.84$ & -1.992 & 0.048 \\
\hline
\end{tabular}

Cohen's effect size $(\mathrm{NGE})=0.360$, Cohen's effect size $(\mathrm{GE})=0.351$ 


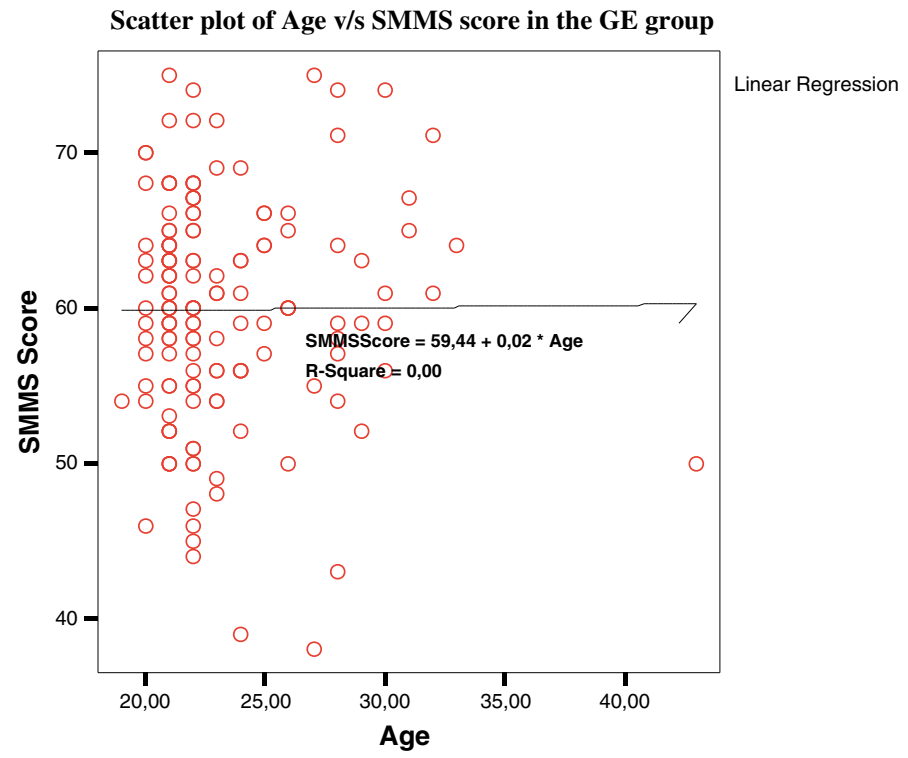

Fig. 1 Scatter plot of age versus SMMS score in the GE group

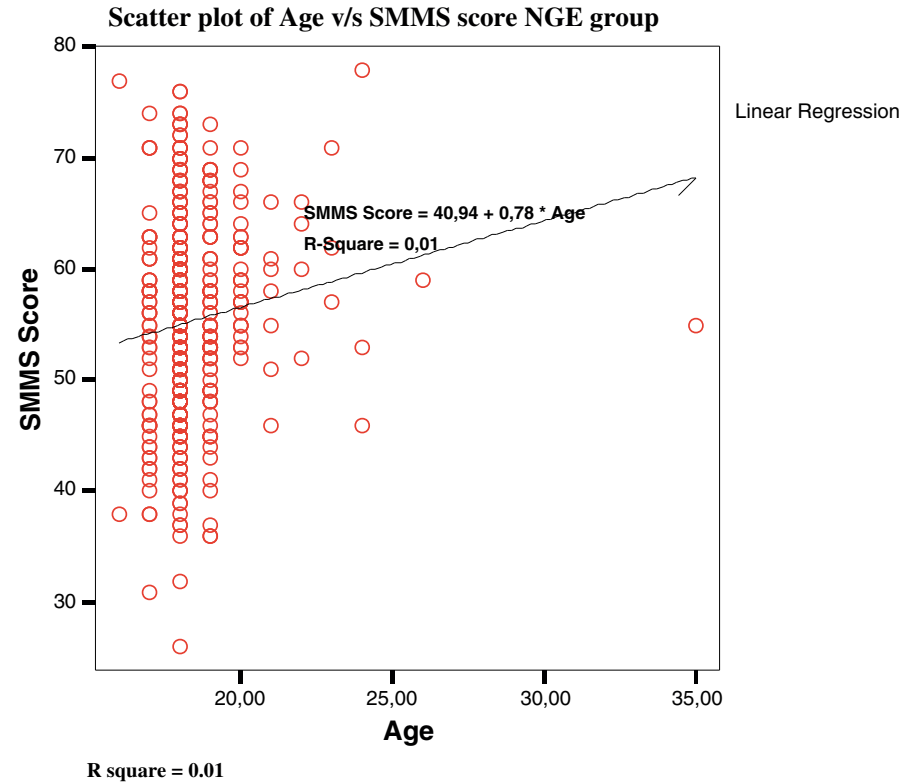

Fig. 2 Scatter plot of age versus SMMS score NGE group

$0.7 \%$. Model 2, in which Maturity was used as a predictor instead of Age, Maturity was able to explain $6.1 \%$ of the variance in the SMMS scores, Gender was able to explain another $1 \%$ and educational background was able to explain another $0.8 \%$. 
Table 5 Model 1 of linear regression analysis

\begin{tabular}{lll}
\hline Model 1 & Adjusted $\mathrm{R}^{2}$ & Standard error of the estimate \\
\hline Age & 0.039 & 8.15 \\
Age & 0.067 & 8.02 \\
Gender & & \\
Age & 0.074 & 7.99 \\
Gender & & \\
Background & & \\
\hline
\end{tabular}

\begin{tabular}{lll}
\hline Model 2 & Adjusted $\mathrm{R}^{2}$ & Standard error of the estimate \\
\hline Maturity & 0.061 & 8.06 \\
$\begin{array}{l}\text { Maturity } \\
\text { Gender }\end{array}$ & 0.071 & 8.02 \\
$\begin{array}{l}\text { Maturity } \\
\text { Gender }\end{array}$ & 0.079 & 7.99 \\
Background & & \\
\hline
\end{tabular}

Table 6 Model 2 of linear regression analysis (age replaced by maturity)

\section{Discussion}

Strength of motivation is not static; it appears to be a dynamic entity as it varies with age and maturity. The present study is a cross-sectional study which shows that strength of motivation increases with age, between the ages of 18 to 24 years. The SMMS scores in this study are in line with the scores obtained by Nieuwhof (Nieuwhof et al. 2004). After the age of about 24 years the strength of motivation is more or less constant (Figs. 1, 2).

The pattern which the maturity of males follows to catch up with maturity of females is not really known, but we assume that it is not linear. Assuming that the amount of catching up decreases as the difference in maturity becomes smaller; we modeled the catch-up as a quadratic function. The merit in using this model is that it allows determining whether there is a true gender difference. With respect to maturation, an evidence-based model by doing a prospective study of the same subject group to determine the change in their strength of motivation for medical school with advancement of age is proposed for the future.

In the present study, age emerged as the single largest predictor of the strength of motivation. This finding is similar to another study, which compared medical students with and without a graduate degree and found age as the most important predictor of outcomes (Wilkinson et al. 2004). The probable cause could be that age and maturity help in making a clear career choice (Wilkinson et al. 2004). Age and maturity have been found to affect learning style and performance in medical school. A study done in Canada found that surface learning style positively correlated with younger age at admission to medical school and negatively correlated with pre-medical university experience (Aaron and Skakun 1999). A study done in the United States, found that students who were judged by the admissions committee to be higher in maturity and motivation were more likely to receive outstanding internship recommendations later (Murden et al. 1978). The cause of these findings may be higher strength of motivation in these students, though it has not been measured in these studies. 
The effect of gender on strength of motivation cannot be explained only on the basis of difference between the maturity of the males and the females, since it persists even after correcting for the confounding effect of the maturity. There exists only one study that has studied the strength of motivation between males and females and it reported no difference. (Hulsman et al. 2007). It is possible that the authors could not find a difference between the strength of motivation in their study whereas our study could, because the sample size in the our was much larger and the response rate of the participants was $91 \%$ as opposed to $76 \%$ in the Hulsman study, which might have not been representative of the whole student population. Other studies have investigated the difference in quality of motivation between males and females. These studies have found that females appear to display lower levels of external control, but higher levels of internal control than males (Cooper et al. 1981). The females are more intrinsically motivated and less extrinsically motivated (Vallerand and Bisonnette 1992). These findings have been replicated in different age group profiles (Vallerand and Fortier 1997; Vallerand and O'Connor 1989). Thus, the gender effect on motivation appears well-supported and seems to extend almost across the life span (Vallerand and Bisonnette 1992). This concords with the finding that female medical students perform better than male medical students and they are more likely to attain an honours degree. (Yates et al. 2009, Fergusson et al. 2002).

The difference between the two groups as far as entrance criteria are concerned is two fold the educational background and pre-entrance selection. For the GE group, the variables educational background and selection are not separable. Every student in the GE group has a bachelor's degree and is selected through a selection procedure. So the effect of these two factors is confounded. Selection by itself could be expected to increase the motivation of the students. The NGE students were selected through weighted lottery depending on their overall grade for the high school diploma. The selection procedure for the GE course was much more difficult and the applicants put in a lot of hard work to prepare for it. This Selection Procedure included Multiple Choice Questionnaire exam, grading of Curriculum Vitae, Scientific Essay, Personal Interview and Patient Interview/Study Sample Assessment Procedure (Oosterveld and ten Cate 2007). SMMS scores did not correlate with the selection procedure scores as a whole (Pearson's correlation coefficient 0.06 ) or any of its subelements. We would expect that the educational background of the students could give them a working knowledge about the science of medicine and time to ponder over whether they find it interesting. This type of in-depth thinking could make the students surer about their choice of career and more motivated. This is in line with the findings of an earlier study (Wilkinson et al. 2004). A study done in Finland established that GE students are a special group within medical students who have greater theoretical and practical commitment to their studies and strong work life orientation as compared to students joining medical school after high school (Kronqvist et al. 2007). In another study, having a prior degree also increased the co-cooperativeness, goal orientation and achieving motive. (Wilkinson et al. 2004). We do not know to what extent experience, as in educational background, affects strength of motivation. Though it has a significant effect, it explains only an extra $0.8 \%$ of the variance in the strength of motivation in our study.

It would be worthwhile to think about the reasons why these GE students did not enter medical school after their high school. One of the possible explanations could be that these students did not have a GPA above 8 to obtain a direct entry into the medical course and did not get chosen in the weighted lottery. It is possible that they tried repeatedly, looked for other means to enter medical school and then tried for the graduate entry course. The other plausible explanation is that their initial choice of career was not medicine, but the interest in medicine was developed somewhere along the way. The third explanation could 
be that there is a group of students who believe that obtaining a bachelor's degree in the life sciences and then joining medicine is the better path to become a doctor than choosing for a traditional medical course after high school. All these explanations point to a more informed career choice and stronger motivation.

Our study has several limitations. The construct validity of the SMMS questionnaire has been explored only in one study, with a low number of respondents (Nieuwhof et al. 2004). More studies are needed to establish firmly the construct validity of this questionnaire. This could be an area for future research.

The variables studied in this study explain only $7.9 \%$ of variance in the strength of motivation for medical school. A major part of the variance remains unexplained. Other factors like personality traits, educational background of parents and nationality need to be evaluated as predictors of strength of motivation.

Age of the students was a confounding factor for assessing the effect of the educational background. But when age was entered into the model, it explained much larger part of the variance in motivation as compared to the educational background.

In our study, it was not possible to separate and measure the effects of educational background and selection. The effects are very much confounded.

The study does not really connect strength of motivation to performance in medical school. This presents an opportunity for further research in this field and it would also be also interesting to determine the change in the strength of motivation over the course of the medical study.

We do not know to what extent we can generalize our findings because the study was carried out in only one institution in The Netherlands. But another study done in New Zealand corroborates our findings (Wilkinson et al. 2004). Nevertheless, we recommend repeating a similar study in other institutions in and outside The Netherlands.

Open Access This article is distributed under the terms of the Creative Commons Attribution Noncommercial License which permits any noncommercial use, distribution, and reproduction in any medium, provided the original author(s) and source are credited.

\section{References}

Aaron, S., \& Skakun, E. (1999). Correlation of students' characteristics with their learning style as they begin medical school. Academic Medicine, 74, 260-262.

Allen, I. (2005). Women doctors and their careers: What now? BMJ, 331(7516), 569-572.

Baum, K. D., \& Axtell, S. (2005). Trends in North American medical education. Keio Journal of Medicine, 54(1), 22-28.

Bickel, J. (2001). Gender equity in undergraduate medical education. A status report. Journal of Women's Health and Gender-Based Medicine, 10(3), 261-270.

Carrothers, R. M., Gregory, S. W., \& Gallagher, T. J. (2000). Measuring emotional intelligence of medical school applicants. Academic Medicine, 75, 456-463.

Cooper, H. M., Burger, J. M., \& Good, T. L. (1981). Gender differences in the academic locus of control beliefs of young children. Journal of Personality and Social Psychology, 40, 562-572.

Ferguson, E., James, D., \& Madeley, L. (2002). Factors associated with success in medical school: systematic review of the literature. BMJ, 324, 952-957.

Heiligers, P. J. M., \& Hingstman, L. (2000). Career preferences and the work-family balance in medicine: gender differences among medical specialists. Social Science and Medicine, 50(9), 1235-1246.

Hulsman, R. L., Van der einde, J. S. J., Oort, F. J., Michels, R. P. J., Casteelen, G., \& Griffioen, F. M. M. (2007). Effectiveness of selection in medical schooll admissions: evaluations of the outcomes among freshmen. Medical Education, 41, 369-377.

Jolles, J., de Groot, R., van Benthem, J., Dekkers, H., de Glopper, C., Uijlings, et al. (2006). Brain lessons. Maastricht: Neuropsych Publishers. Retrieved June 30, 2009, From http://www.hersenenenleren.nl/ 2006Jolles_BrainLessons.pdf. 
Kronqvist, P., Mäkinen, J., Ranne, S., Kääpä, P., \& Vainio, O. (2007). Study orientations of graduate entry medical students. Medical Teacher, 29, 836-838.

Marley, J., \& Carman, I. (1999). Selecting medical students: A case report of the need for change. Medical Education, 33, 455-459.

Marshall, W. A., \& Tanner, J. M. (1970). Variations in the patterns of prepubertal changes in boys. Archives of disease in childhood, 45, 13-23.

Murden, R., Galloway, G. M., Reid, J. C., \& Colwill, J. M. (1978). Academic and personal predictors of clinical success in medical school. Journal of Medical Education, 53, 711-719.

Nieuwhof, M. G. H., Rademakers, J. J. D. M., Kuyvenhoven, M. M., Soethout, M. B. M., \& ten Cate, O. Th. J. (2005). Students' conceptions of the medical profession: An interview study. Medical Teacher, 27(8), 709-714.

Nieuwhof, M. G. H., ten Cate, O. Th. J., Oosterveld, P., \& Soethout, M. B. M. (2004). Measuring strength of motivation for medical school (Electronic version). Medical Education Online, 9, 16. Retrieved May 28, 2009, from http://www.med-ed-online.org.

Oosterveld, P., \& ten Cate, O. Th. J. (2007). Generalizability of a study sample assessment procedure for entrance selection for medical school. Medical Teacher, 26(7), 635-639.

Phillips, S. P., \& Austin, E. B. (2009). The feminization of medicine and population health. JAMA, 301(8), $863-864$.

Prideaux, D., \& McCrorie, P. (2004). Models for the development of graduate entry medical courses: Two case studies. Medical Education, 38, 1169-1175.

Pujol, J., Vendrell, P., Junque, C., Marti-Vilalta, J. L., \& Capdevila, A. (1993). When does human brain development end? Evidence of corpus callosum growth up to adulthood. Annals of Neurology, 34, 7175.

Quality Assurance Netherlands Universities (QANU) Report for medical schools. (2004). Retrieved June 30, 2009, from: http://www.qanu.nl/comasy/uploadedfiles/GeneeskundeRapportwebsite.pdf.

Reed, R. B., \& Stuart, H. C. (1959). Patterns of growth in height and weight from birth to eighteen years of age. Paediatrics, 24, 904-921.

Steinberg, L. (2005). Cognitive and affective development in adolescence. Trends in Cognitive Sciences, 9(2), 69-74.

Steinberg, L., \& Cauffman, E. (1996). Maturity of judgment in adolescence: Psychosocial factors in adolescent decision making. Law and Human Behavior, 20(3), 249-272.

Tanner, J. M., Whitehouse, H. R., Marubini, E., \& Resele, L. F. (1976). The adolescent growth spurt of boys and girls of the harpenden growth study. Annals of Human Biology, 3(2), 109-126.

ten Cate, O. Th. J. (2007). Medical education in the Netherlands. Medical Teacher, 29, 752-757.

Underwood, M. J., Thompson, M., \& McCaskie, A. (1990). Insight of first-year medical students into their future working conditions. Medical Education, 24(3), 210-211.

Vallerand, R. J., \& Bisonnette, R. (1992). Intrinsic, extrinsic and amotivational styles as predictors of behavior: A prospective study. Journal of Personality, 60(3), 599-620.

Vallerand, R. J., \& Fortier, M. S. (1997). Self-determination and persistence in a real-life setting. Toward a motivational model of high school dropout. Journal of Personality and Social Psychology, 72(5), 1161-1176.

Vallerand, R. J., \& O'Connor, B. P. (1989). Motivation in the elderly: A theoretical framework and some promising findings. Canadian Psychology, 30, 538-550.

Velden, L.F.J., Hingstman, L., Heiligers, P.J.M. \& Hansen, J. (2008). Toenemed percentage vrouwen in de geneeskunde: verleden, heden en toekomst (Increasing proportion of women in medicine: past, present and future). Ned. Tijdschr. Geneeskd. (Dutch Journal of Medicine), 152(40), 2165-2171.

VSNU (Association of Universities, The Netherlands). (2008, October 9). Technische University, Delft. Talent uitdagen - Studiesucces in de Bachelor fase (Talent Challenge-Student Success in the Bachelor phase). Retrieved June30, 2009, from http://www.vsnu.nl/web/file?uuid=82c51d91-88c9-44f8-870db05191be9afc\&owner=6fdf361c-e6d9-4412-a0c3-fa860071195e.

Westenberg, P.M. (2008). De Jeugd van Tegenwoordig! (Plenary 433rd Annual address at the opening of the academic year of University of Leiden in The Netherlands). Retrieved June 11, 2009, from Leiden University Website: http://www.leidenuniv.nl/tekstboekjes/content_docs/oratie_westenberg.pdf.

Wilkinson, T. J., Wells, J. E., \& Bushnell, J. A. (2004). Are differences between graduates and undergraduates in a medical course due to age or prior degree? Medical Education, 38, 1141-1146.

Yates, J., Smith, J., James, D., \& Ferguson, E. (2009). Should applicants to Nottingham University medical school study a non-science A-level? A cohort study. BMC Medical Education, 9, 5-13. 